\title{
RELACIÓN ENTRE LA RESILIENCIA Y LA CALIDAD DE VIDA EN ESTUDIANTES UNIVERSITARIOS DE CAJAMARCA
}

\section{Relationship between Resilience and the Quality of Life in university students of Cajamarca}

Esthefani Fiorella Chávez Hinostroza*

\begin{abstract}
Resumen
Este estudio tuvo como objetivo determinar la relación entre la resiliencia y la calidad de vida de los estudiantes de una universidad pública en Cajamarca. Se evaluó un grupo de 369 estudiantes de ambos sexos, de diferentes ciclos y pertenecientes a todas las facultades de la institución. Los instrumentos de medición fueron la Escala de Resiliencia de Wagnild \& Young y la Escala de Calidad de Vida de Olson \& Barnes. Los resultados muestran una relación significativa directa entre la resiliencia y la calidad de vida, así como de la resiliencia y las dimensiones de calidad de vida: Hogar y bienestar económico; Amigos, vecindario y comunidad; Vida familiar y familia extensa; Educación y ocio; Medios de comunicación; Religión y Salud. Se concluye que la resiliencia es un factor determinante para lograr bienestar que redunda en la calidad de vida de los estudiantes coadyuvando a su desarrollo en cada dominio del ciclo vital.
\end{abstract}

Palabras clave: Resiliencia, calidad de vida, estudiantes universitarios, adaptabilidad, bienestar

\begin{abstract}
This study aimed to determine the relationship between resilience and the quality of life of students of a public university in Cajamarca. A group of 369 students of both sexes, of different cycles and belonging to all the faculties of the institution were evaluated. The measuring instruments were the Resilience Scale of Wagnild \& Young and the Quality of Life Scale of Olson \& Barnes. The results show a significant direct relationship between resilience and quality of life, as well as resilience and the dimensions of quality of life: Home and economic well-being; Friends, neighborhood and community; Family life and extended family; Education and leisure; Media; Religion and Health. It is concluded that resilience is a determining factor for the achievement of well-being which results in the quality of life of students contributing to their development in each domain of the life cycle.
\end{abstract}

Keywords: resilience, quality of life, university students, adaptability, well-being

\footnotetext{
* Artículo basado en la tesis de Licenciatura sustentada por la autora

* Psicóloga fiorela010393@hotmail.com

ORCID https://orcid.org/0000-0002-0583-3867
} 


\section{INTRODUCCIÓN}

La existencia humana compromete para cada individuo una suma de circunstancias críticas, cuyos estresores pueden perturbar la estabilidad psicológica, afectiva y conductual ante su estado de vida (GarcíaAlandete, 2016; Theis, 2003; Turcaz \& Rubio, 2015). En este contexto un individuo puede adoptar un comportamiento disfuncional o reaccionar con mayor fuerza para afrontar sus carencias vitales, es decir, forjar resiliencia (Hu, Zhang, \& Wang, 2015; Sambrano, 2011; Yehuda et al., 2013). La problemática propia de la vida en sociedad repercute negativamente en una nación en pleno, por lo que el enfoque de resiliencia permite evitar pérdidas humanas, disminuir el gasto público y desigualdades sociales para combatir la pobreza a fin de promover el desarrollo social sostenible (Comisión Europea, 2012). Sin embargo, cada grupo humano asume una función propia para el bienestar colectivo (Pérez-Escoda, 2013), ¿qué rol cumplen los jóvenes?, ¿pueden los jóvenes desarrollar resiliencia para mejorar sus condiciones de vida?, ¿hay un vínculo directo entre la resiliencia y la calidad de vida para el fomento del potencial humano de esta población en etapa universitaria?

La dinámica articulada entre la resiliencia y la calidad de vida, se afianza a través del proceso académico-formativo de los jóvenes en aras de un desenvolvimiento integral en la vida como contempla la Agenda 2030 de la Organización de las Naciones Unidas (ONU, 2015) que considera a la educación de calidad un objetivo principal para alcanzar sostenibilidad global. En el Perú, el índice de la educación ajustado por la desigualdad es 0.536 y hay un $20.3 \%$ de desigualdad en la educación, puntuación elevada entre los países de la región; la tasa bruta de matriculación terciaria en el periodo 2010-2015 es $41 \%$, mientras la participación en la fuerza de trabajo de varones y mujeres de quince años a más, está dada por un $82.6 \%$ y $65.7 \%$ respectivamente, ante un índice de pobreza multidimensional de 0.043 (Programa de las Naciones Unidas para el Desarrollo [PNUD], 2016) que requiere una respuesta inmediata. Dichas carencias en materia educativa podrían limitar la superación de los jóvenes universitarios, condicionando su capacidad de afrontamiento en la vida y por ende su autorrealización. En consecuencia, amerita fomentar rasgos pro-resilientes para favorecer su desarrollo personal, así desde el ámbito educativo facilitar las condiciones básicas de desarrollo humano que los predispongan a calidad de vida (Fraga, 2015; Pérez-Escoda, 2013; Villasmil, 2010).

En Cajamarca, solo el 7.8\% de la población cuenta con educación superior y los jóvenes de 22-24 años tienen una tasa de conclusión universitaria de 13\% (Ministerio de Educación del Perú, 2017), factores que afectan negativamente la participación económica e inclusión laboral de los jóvenes, lo cual incrementa las brechas sociales que los dividen generándoles un ejercicio ciudadano limitado. Por tanto, la resiliencia constituye una capacidad elemental para lidiar con los riesgos a los que expone la adversidad y así, consolidar a diversos niveles la calidad de vida (Seligman \& Czikszentmihalyi, 2000), fortaleciendo el proceso educativo de los estudiantes como un factor clave para el crecimiento social (Organización para la Cooperación y el Desarrollo Económico [OCDE], 2016).

Entre las teorías que abordan el binomio resiliencia-calidad de vida y su interacción con el ámbito educativo, Omar, Paris, Uribe, Ameida, \& Aguiar (2011) proponen un modelo de resiliencia que comprende el optimismo, el sentido del humor, la inteligencia emocional, las emociones positivas y el bienestar subjetivo; el cual sugieren, permitirá redimensionar las fortalezas de los jóvenes para enfrentar situaciones complejas que incidan en el enriquecimiento de su potencial. Por otro lado, Saavedra, Salas, Cornejo, \& Morales (2015) plantean un enfoque de la educación a partir de la calidad de vida para los estudiantes, el cual contribuya al aprendizaje en una forma de vida orientada al logro de la felicidad personal; donde la apertura, participación, flexibilidad y diálogo social dirijan a los jóvenes a su desarrollo en equilibrio con las capacidades de una sociedad proresiliente.

Las investigaciones internacionales previas sobre el tema en cuestión, reportan que a mayores experiencias vitales negativas se producirá menos bienestar psicológico personal $(r=-.23$ a -52$)$, siendo la resiliencia mediadora entre la relación de ambos (Faircloth, 2017); que la resiliencia tiene un rol significativo en la determinación de calidad de vida 
en general y sus dimensiones ( $p>0.001)$, enfatizando la importancia de agenciar a los estudiantes de mayor conocimiento en el campo de la resiliencia para obtener mejor calidad de vida (Bastaminia, Rezaei, Rezaei, \& Tazesh, 2016); asimismo, que los estresores percibidos en la vida académica universitaria incidirán en menor satisfacción con la vida, pero evocarán más recursos resilientes como estrategia de gestión del estrés (Cazan \& Truta, 2015); se presentan niveles altos de autoestima, asertividad y motivación al logro, niveles medios de afiliación y una baja motivación al poder como rasgos que favorecen el desarrollo de resiliencia en los universitarios para alcanzar calidad de vida (Villasmil, 2013); los estudiantes universitarios que perciben su calidad de vida como adecuada, evidencian mayores factores protectores familiares $(r=.22, \mathrm{p}<0.01)$ y sociales $(r=.22, \mathrm{p}<$ $0.01)$ del potencial resiliente; así también, presentan menores factores de riesgo personales $(\mathrm{r}=-.20, \mathrm{p}<$ $0.01)$, familiares $(r=-.20, \mathrm{p}<0.01)$, y sociales $(r=$ $-.24, \mathrm{p}<0.01$ ) de resiliencia (Mikulic et al., 2011).

En el Perú, no hay antecedentes sobre la relación específica entre resiliencia y calidad de vida en la población universitaria; sin embargo, entre los estudios más representativos, Salazar (2017) señala que la expresión de un comportamiento optimista pese a la dificultad contribuye a la felicidad en la vida $(\mathrm{r}=$ .545, p < .001); López, Guevara, \& Quinteros (2016) indican que la religiosidad se asocia con la resiliencia influyendo en el bienestar personal $(r=.598)$, mientras Cáceres \& Ccorpuna (2015) refieren que la resiliencia conducirá favorablemente a superar los estresores académicos para obtener satisfacción individual. En Cajamarca, tampoco se cuentan con referencias previas sobre el tema en particular, la investigación de mayor vinculación pertenece a Huamán \& Jáuregui (2015) quienes reportaron que los jóvenes con baja ideación suicida muestran resiliencia en un nivel moderado en un $67,4 \%$. Es decir, más resiliencia conlleva a menor índice de ideación suicida para salvaguardar la propia vida manteniéndola en perspectiva por sobre la adversidad, y por ende acceder a calidad de vida.

Ante la ausencia de estudios aplicados a la realidad local sobre la vinculación concreta de actitudes resilientes para alcanzar una calidad de vida positiva en los estudiantes universitarios como elemento clave en la productividad de un país, surge este trabajo para contar con un cuerpo teórico específico que guíe futuras intervenciones en el campo de la psicología positiva para incrementar el desarrollo del capital humano en la ciudad de Cajamarca.

\section{MÉTODO}

\section{Diseño}

Esta investigación tiene un diseño transversal al ser elegida la muestra de estudio y recabar información de ella solo en un periodo de tiempo determinado (Shaughnessy, Zechmeister, \& Zechmeister, 2012); asimismo, es de tipo descriptivo-correlacional, porque se identificó y midió dos variables, recopilando información sobre cada una de ellas para analizar su relación estadística (Baker, 2017).

\section{INSTRUMENTOS}

\section{Resiliencia}

La resiliencia fue medida a través de la Escala de Resiliencia de Wagnild \& Young (1993). Evalúa las dimensiones de: Ecuanimidad, Sentirse bien solo, Confianza en sí mismo, Perseverancia y Satisfacción personal. La escala está compuesta por dos factores: el Factor I, denominado Competencia personal y el Factor II, denominado Aceptación de uno mismo y de la vida. Está conformada por 25 ítems que se puntúan en una escala de 7 puntos, donde 1 es en desacuerdo y un máximo de acuerdo es 7 , mayores puntajes son indicadores de mayor resiliencia. En el Perú, este instrumento ha sido adaptado por Novella (2002), obteniendo una confiabilidad de .88 mediante el coeficiente alfa; la correlación item-test mostró que cada dimensión de la escala es confiable y se determinó que este instrumento mide apropiadamente el constructo resiliencia como valor absoluto.

\section{Calidad de vida}

La calidad de vida fue medida a través de la Escala de Calidad de Vida de Olson \& Barnes (1982). Evalúa las dimensiones de: Hogar y bienestar económico; Amigos, vecindario y comunidad; Vida familiar y familia extensa; Educación y ocio; Medios de comunicación; Religión y Salud. Está compuesta 
por 24 ítems que se puntúan en una escala de 5 puntos, donde 1 es insatisfecho y un máximo de satisfacción es 5 , mayores puntajes son indicadores de mayor calidad de vida. En el Perú, este instrumento ha sido adaptado por Grimaldo (2003), obteniendo una confiabilidad de .86 mediante el coeficiente alfa; se halló el $56 \%$ de la varianza de los ítems, un indicador que confirma la estructura teórica del instrumento; en cuanto al índice de homogeneidad, todos los ítems obtienen puntajes superiores al margen acceptable, manifestación de la discriminación conjunta de los ítems en el mismo sentido que el instrumento total.

\section{Ficha sociodemográfica}

Este instrumento recopiló datos de la población en estudio correspondientes a la información de sus condiciones de vivienda, descriptores económicos, educativos, académicos y algunos aspectos adicionales para contar con una noción situacional de la muestra en análisis.

\section{Consentimiento informado}

Este instrumento permitió brindar alcances sobre la propuesta, beneficios, costos e incentivos, lineamientos de confidencialidad, uso de la información obtenida y derechos con los que cuentan al participar, para proporcionar su autorización como sujetos humanos en investigación.

\section{PARTICIPANTES}

La muestra de estudio estuvo conformada por 369 estudiantes de la Universidad Nacional de Cajamarca, cuya subpoblación fue distribuida según un muestreo probabilístico por conglomerados. Del total de evaluados, destaca el $61,8 \%$ que tenían de 16 a 20 años y el $34,7 \%$ de 21 a 25 años; $41,5 \%$ eran mujeres y $58,5 \%$ eran varones; de la mayoría, $33,6 \%$ tenía un ingreso monetario familiar de 1000 a 1999 soles y $27,1 \%$ un ingreso monetario familiar de 2000 a 2999 soles. Solo el 8,7\% autofinanciaba sus estudios y el $84 \%$ tenía una motivación académica intrínseca por sobre la extrínseca; todos los estudiantes profesaban una religión y solo $16,3 \%$ del total poseía habilidades diferentes. Se evaluaron al conjunto de facultades considerando una carrera por cada una de ellas y algunos ciclos por sorteo, los cuales se detallan a continuación:

Tabla 1

Distribución de estudiantes de una universidad pública de Cajamarca según la facultad, carrera y ciclos a los que pertenecieron

\begin{tabular}{llcc}
\hline Facultades & \multicolumn{1}{c}{ Carreras } & Ciclos & Porcentaje \\
\hline Ingeniería & Ingeniería de Sistemas & III, V, VI & $10,6 \%$ \\
Medicina Humana & Medicina Humana & I, V & $10,3 \%$ \\
Ciencias Agrarias & Ingeniería de Industrias Alimentarias & V, IX, X & $10,3 \%$ \\
Ciencias de la Salud & Enfermería & I, IX & $10 \%$ \\
Ciencias Económicas, Contables y Administrativas & Contabilidad & III, VII, IX & $10 \%$ \\
Ciencias Pecuarias & Ingeniería Zootecnista & I, III, IX & $9,8 \%$ \\
Ciencias Sociales & Turismo y Hotelería & III, VI & $9,8 \%$ \\
Ciencias Veterinarias & Medicina Veterinaria & III, VII, IX,X & $9,8 \%$ \\
Derecho y Ciencias Políticas & Derecho & V, VII & $9,8 \%$ \\
Educación & Educación & II, VII, IX, X & $9,8 \%$ \\
\hline
\end{tabular}




\section{PROCEDIMIENTO}

En primera instancia se determinó el tema de trabajo considerando las implicancias teóricoprácticas ya descritas para atender a una problemática representativa en la psicología; seguidamente se establecieron los fundamentos teóricos que regirían el estudio y se seleccionaron las pruebas psicológicas a usar. Luego, se realizó una adaptación lingüística de la Escala de Resiliencia y de la Escala de Calidad de Vida a la realidad local, así como la validación de dichos instrumentos por jueces expertos; ambos procesos evidenciaron que los reactivos que miden cada uno de los contructos en investigación son apropiados para su evaluación en este contexto. Posteriormente, se gestionó el permiso correspondiente con las autoridades pertinentes de la universidad nacional para la evaluación de los estudiantes; durante la aplicación de los instrumentos se dio a conocer de qué trataban y el objetivo de cada uno, monitoreando la tarea y resolviendo las dudas presentes; cabe señalar que en principio todos los participantes firmaron el consentimiento informado previamente descrito para cumplir con los lineamientos de ética establecidos por la institución de afiliación del presente estudio, así como la normativa vigente a nivel nacional e internacional para la investigación psicológica.

\section{ANÁLISIS DE DATOS}

El procesamiento estadístico de la información recabada se realizó a través del programa estadístico IBM SPSS Statistics versión 24. Inicialmente, se calculó la confiabilidad de los intrumentos aplicados mediante el coeficiente alfa, siendo para la Escala de Resiliencia de Wagnild \& Young de .842 y para la Escala de Calidad de Vida de Olson \& Barnes de .86. Luego, se evaluó la normalidad de los datos obtenidos por medio de la prueba Kolmogorov-Smirnov, la cual determinó la aplicación del coeficiente de correlación de Pearson al presentarse una distribución normal. Es preciso mencionar que solo se midió la correlación entre la variable resiliencia como valor absoluto respecto de la variable calidad de vida y sus dimensiones debido a que existe mayor información en comparación de las dimensiones de resiliencia y su relación con la calidad de vida.

\section{RESULTADOS}

En la tabla 2 se puede observar que hay una relación significativa directa entre la resiliencia y la calidad de vida $(\mathrm{r}(369)=, 456 ; \mathrm{p}<.01)$ de los estudiantes universitarios.

Tabla 2

Correlación entre Resiliencia y Calidad de Vida

\begin{tabular}{lcc}
\hline & Resiliencia & Calidad de vida \\
\hline Correlación de Pearson &, $456^{* *}$ \\
Sig. (bilateral) &, 000 \\
N & 369 \\
\hline
\end{tabular}

**. La correlación es significativa en el nivel 0,01 (bilateral).

En la tabla 3 se puede observar que hay una relación significativa directa entre la resiliencia y las dimensiones de calidad de vida: Hogar y bienestar económico $(r(369)=, 324 ; p<.01)$; Amigos, vecindario y comunidad $(r(369)=, 350 ; p<.01)$; Vida familiar $\mathrm{y}$ familia extensa $(r(369)=, 352 ; p<.01)$; Educación y ocio $(r(369)=, 323 ; p<.01)$; Medios de comunicación $(r(369)=, 283 ; p<.01)$; Religión $(r(369)=, 238 ; p<$ $.01)$ y Salud $(r(369)=, 218 ; p<.01)$ de los estudiantes universitarios.

Tabla 3

Correlación entre Resiliencia y las dimensiones de Calidad de Vida

\begin{tabular}{lcc}
\hline & $\begin{array}{c}\text { Resiliencia } \\
\text { Correlación } \\
\text { de Pearson }\end{array}$ & Sig. (bilateral) \\
\hline Hogar y bienestar económico &, $324 * *$ &, 000 \\
Amigos, vecindario y comunidad &, $350^{* *}$ &, 000 \\
Vida familiar y familia extensa &, $352^{* *}$ &, 000 \\
Educación y ocio &, $323^{* *}$ &, 000 \\
Medios de comunicación &, $283^{* *}$ &, 000 \\
Religión &, $238^{* *}$ &, 000 \\
Salud &, $218^{* *}$ &, 000 \\
\hline
\end{tabular}

**. La correlación es significativa en el nivel 0,01 (bilateral). 


\section{DISCUSIÓN}

La resiliencia contempla una posición alternativa a los patrones de afrontamiento tradicionales en el desarrollo vital de cada individuo, que implica realizar un juicio crítico de cada experiencia para analizarla y en último término, tomar acción para superarla. Así, en el ámbito educativo ser resiliente conlleva asumir responsabilidades que permitan un buen redimiento académico y por ende una vida satisfactoria asumiendo un enfoque personal positivo y de la comunidad, lo cual repercuta en el bienestar de sus miembros, como en la región Cajamarca para fortalecer los actuales indicadores de desarrollo humano considerando a la educación un pilar esencial que mediante la resiliencia y su asociación con la calidad de vida contribuya a la mejora de la comunidad.

Respecto del objetivo general establecido, se confirma que hay una relación significativa directa entre la resiliencia y la calidad de vida de los estudiantes de una universidad pública de Cajamarca, corroborándose la hipótesis general. Estudios previos señalan que la resiliencia constituye un mecanismo dinámico producto de la interacción persona-contexto (Pereira, 2007) que promueve resistencias sostenibles ante las crisis para considerarlas en un sentido más amplio y profundo (Asociación Americana de Psicología [APA], 2014) en aras de lograr una reintegración individual (Yehuda et al., 2013), que permita la adopción de un comportamiento funcional ante las limitaciones presentes. Puesto que la resiliencia es un rasgo personal cuyos atributos son inherentes a la expresión de actitudes de vida positivas (Connor \& Davidson, 2003; Hu et al., 2015; Ong, Bergeman, Bisconti, \& Wallace, 2006; Wagnild \& Young, 1993), es una función que facilita la recuperación individual para dotar las experiencias de un caracter más significativo (Harvey \& Delfabbro, 2004; Hu et al., 2015; Masten, 2001) y un proceso para adaptarse activamente conjugando la capacidad de protección y la de reacción para recuperarse frente a la presión (Fergus \& Zimmerman, 2005; Hu et al., 2015; Luthar, Cicchetti, \& Becker, 2000).

Por lo tanto, es un concepto multidimensional determinante para la realización personal, pues en base a la capacidad para lidiar con las dificultades, se podrá superar con éxito las barreras y sobreponerse. Así, se relaciona con la calidad de vida, ya que una persona resiliente logra rehacerse, reaprender y reactivarse, equilibrando las propias demandas con las del entorno para satisfacer diversos dominios de la vida y dar valor a la existencia (Leipold \& Greve citados en Fletcher \& Sarkar, 2011; Olson \& Barnes, 1982; Unión Europea, 2015); confirmándose estudios previos, que evidencian que los individuos resilientes pueden transformar sus debilidades para cumplir un proyecto de vida positivo más allá de la adversidad (Bastaminia et al., 2016; Cazan \& Truta, 2015; Faircloth, 2017; González \& Artuch, 2014; Mikulic et al., 2011; Salazar, 2017; Villasmil, 2013). La resiliencia respecto a la calidad de vida apertura nuevas rutas para reinventarse y cuestionar lo establecido para forjar nuevos paradigmas y así, y solo así reconstruirse y crecer como individuo para redefinir la realidad y asumir un rol activo ante los propios límites, puesto que si se es resiliente se podrá lograr una vida con calidad.

Respecto a los objetivos específicos establecidos, se confirma que hay una relación significativa directa entre la resiliencia y las dimensiones de calidad de vida: Hogar y bienestar económico, referida a la capacidad económica de la familia; Amigos, vecindario y comunidad, concerniente a la relación que mantiene el individuo con el grupo de pares; Vida familiar y familia extensa, alusiva a la relación de afecto con el grupo de apoyo primario; Educación y ocio, referente a las oportunidades educativas y recreativas del individuo; Medios de comunicación, concerniente al grado de satisfacción con dichos medios de contacto masivo; Religión, relativa a la vida religiosa que sigue la familia y Salud, alusiva al nivel de salud en general de todos los miembros del hogar de los estudiantes de una universidad pública de Cajamarca, corroborándose las hipótesis específicas; hallazgos discutidos uno a uno a continuación.

En primer lugar, los jóvenes resilientes se caracterizan por contar con redes de apoyo familiar (Gómez, 2010) para perseguir un objetivo mutuo, sea tangible o intangible, frente a las vulnerabilidades existentes, tal como el 91,3\% de estudiantes evaluados que reportaron recibir apoyo externo para financiar sus estudios. Realizan una toma de decisiones orientada 
a soluciones efectivas a los problemas (Ballesteros, 2015) en función de sus prioridades para obtener los recursos que garanticen un desarrollo de vida adecuado. Asimismo, los jóvenes resilientes actúan con determinación (Fraga, 2015) para adecuarse y evitar factores de riesgo como una situación laboral inactiva, que derive en sentimientos de minusvalía y en no contar con ingresos económicos (GonzálezArratia \& Valdez, 2013) para su propio sustento y el de su hogar. Sin embargo, al recibir la asistencia de los demás, como la familia, se cuenta con un importante factor de protección debido a la incondicionalidad afectiva expresa que fortalece la autoeficacia para mantenerse firmes en cumplir las responsabilidades asumidas (Calderón, 2016) a fin de lograr bienestar económico para cubrir las necesidades vitales (PNUD, 2016) y tener una vida estable en su conjunto desde el medio familiar.

En segundo lugar, la resiliencia se aplica a nivel micro y macrosocial cuando el apoyo de los pares y del estado que promueve las capacidades de sus miembros conduce a un autodescubrimiento continuo sabiendose sostenido (Michaud citado en Cyrulnik, 2002); asimismo, los factores sociopolíticos que impulsan la resiliencia brindan los instrumentos para gestionarla en beneficio de la comunidad (Notter, McTavish, \& Shamah, 2008), y así delimitar en positivo los índices de calidad de vida en el colectivo. Los estudiantes resilientes perciben como estímulo motivacional tanto a los amigos como a las instituciones y su influencia en el fomento de iniciativas pro-jóvenes (Torres \& Ruiz, 2013), al interior de diversos grupos sociales se producen vínculos que inciden en la conexión emocional con el entorno (APA, 2014), la contención psicoafectiva de otros individuos contribuye a una vida satisfactoria (Pérez-Escoda, 2013) y la procedencia cultural, el medio de creencias y costumbres en las que una persona se desenvuelve determinan su bienestar (Monardes, González-Gil, \& Soto-Pérez, 2011) resultante de la asunción de un rol pro-resiliente. La medida de bienestar poblacional se rige según las conexiones interpersonales, el compromiso cívico y la buena gobernanza (OECD, 2017), indicadores de integridad ciudadana para viabilizar resiliencia $y$, por ende, calidad de vida. En la interacción social con la comunidad se genera un estrecho nexo relacional que influye en el nivel de afrontamiento conjunto evocando resiliencia ante las amenazas como agente facilitador de calidad de vida óptima.

En tercer lugar, la constitución de un grupo sólido que posee vínculos consanguíneos donde se han forjado relaciones de confianza y empatía recíproca confiere la seguridad para autorregularse ante cada contratiempo y enfrentar la realidad presente (Grotberg, 2006). La crianza asertiva de parte de una figura con quien unen lazos de apego permite desarrollar un ciclo de vida equilibrado (Barudy \& Dantagnan, 2011) para reforzar rasgos resilientes en la estructura familiar. Asimismo, el simbolismo de las relaciones interindividuales se distigue según las situaciones vivenciadas por cada uno posibilitándoles un enfoque más positivo (Obando, Villalobos, \& Arango, 2010) en aras de bienestar; y la tolerancia de los propios impedimentos y del ambiente conlleva a cultivar una visión optimista para redimensionar las carencias y sobresalir (APA, 2014) promoviendo resiliencia y satisfacción personal para favorecer la funcionalidad familiar. Contar con las condiciones materiales necesarias para la subsistencia en la vida familiar influye en el potencial resiliente de uno mismo y su familia (PNUD, 2014); repercutiendo en la calidad de vida y desarrollo sustentable del medio en su conjunto (Cantú-Martínez, 2015).

En cuarto lugar, los estudiantes resilientes mantienen un firme compromiso en el cumplimiento de sus asignaciones académicas, traducido en la certeza de sus habilidades y competencias para llevar a cabo un desempeño efectivo (Villasmil, 2010), evidenciado en la motivación intrínseca que el conjunto de los estudiantes evaluados reportó en un $84 \%$ como incentivo para cursar sus estudios. El dinamismo de un clima institucional positivo entre compañeros, docentes y autoridades está asociado a una postura resiliente y su correlación significativa con un buen rendimiento de los educandos (Gallesi \& Matalinares, 2012). Las estrategias de resiliencia alineadas a la formación de los jóvenes en etapa educativa incrementan su autoeficacia en cada tarea por sobre su complejidad (Villasmil, 2013), incidiendo en la disminución de aquellos factores que interfieren en su aprendizaje (Cáceres \& Ccorpuna, 2015) y consecuente inserción en el campo laboral (Alonso-Aldana, BeltránMárquez, Máfara-Duarte, \& Gaytán-Martínez, 2016); por lo tanto, los estudiantes resilientes se predisponen 
a calidad de vida. La obtención de logros y destacar al interior del círculo académico proporciona un referente para proyectarse a futuro en aras de un desenvolvimiento idóneo en cualquier ámbito de la vida (Velasco, Suárez, Córdova, Luna, \& Mireles, 2015). A su vez, el perfil del docente, se configura como el agente principal para transmitir a los jóvenes un carácter resiliente, impulsando destrezas creativas y autodisciplina (Aguaded \& Almeida, 2016), para cimentar una vida estable, cuya calidad deviene de su condición instrumental (Nava, 2012); contribuyendo a atender los actuales índices de educación reportados en la región Cajamarca (Ministerio de Educación del Perú, 2017) que requieren posicionarse como punto focal para la mejora educativa local.

En quinto lugar, los medios de comunicación son cruciales en la formación de la cultura, que por medio de la resiliencia empodera a los ciudadanos para resignificar los prejuicios hacia ciertos grupos sociales, de manera que se comprenda, desde una perspectiva sistémica-ecológica, cómo al interior de una comunidad se determina en el curso de la vida el efecto de mantener una actitud resiliente para alcanzar calidad de vida general (Ruiz-Román, Calderón-Almendros, \& Juárez, 2017) repercutiendo en diversos dominios del ciclo vital de la población universitaria joven. Dichos medios tienen por objetivo que los individuos desarrollen una conciencia crítica para el cambio social, fuente importante resiliencia (Obando, Villalobos, \& Arango, 2010), a fin de modificar patrones desdaptativos de conducta que incidan en una vida estable. Tener la oportunidad de manifestarse y brindar atención a los demás para llevar a cabo un intercambio compartido es otra fuente sustancial de resiliencia en la interacción humana (Barudy \& Dantagnan, 2011), aspecto central en los medios de comunicación masivos. Aquellos sistemas de apoyo externos a la familia son factores protectores de los riesgos que exponen los estresores ambientales (Mateau, García, Gil, \& Caballer, 2009), la accesibilidad a los servicios sociales para atender cualquier eventualidad promueve la resiliencia (Leiva, Pineda, \& Encina, 2013), como el establecimiento de contacto con medios de comunicación para custodiar el bienestar colectivo. Los enfoques referidos a derechos humanos, criterios interculturales y un enfoque generacional (ONU, 2016) persiguen el desarrollo sostenible, temáticas actuales que abordan los medios de comunicación contribuyendo a una sociedad más resiliente y con mayor calidad de vida.

En sexto lugar, los estudiantes resilientes sostienen creencias arraigadas en la finalidad y fundamento de la vida (SENA, 2010), avizoran el futuro con esperanza y desarrollan una visión positiva de su contexto de vida pese a las desavencias surgidas (Everly, McCormack, \& Strouse, 2012). Los jóvenes resilientes reflejan una espiritualidad que les concede autoconfianza y una fe firme por sobre el grado de dificultad de sus experiencias (Hernández-Holguín, Sánchez, Páez, \& Montoya-Vásquez, 2016), reflejado en todos los estudiantes en estudio que reportaron profesar una religión; asimismo, mantienen una sólida convicción para descubrir en sí mismos mayores fortalezas ante sus carencias (APA, 2014) y lograr bienestar. También, planifican sus responsabilidades adquiridas con una respuesta optimista desde la cotidianidad (Instituto de HeartMath, 2014), resultado de asumir una postura de fe que redunda en cada área de la vida. La religión brinda los cimientos para realizar con libertad acciones decisivas para una calidad de vida sustantiva, con implicancias éticas y externas a la persona (Nava, 2012); por tanto, está asociada a la resiliencia como evidencian estudios previos (López, Guevara, \& Quinteros, 2016), y en ese sentido, a la consolidación de mejor calidad de vida.

En séptimo lugar, las personas resilientes aceptan sus impedimientos como incentivo ante graves enfermedades o accidentes para recuperarse y salir fortalecidas (Michaud citado en Cyrulnik, 2002) incluso frente a estados de discapacidad (Federación Española de Síndrome de Down, 2013), expreso en el $16,3 \%$ de estudiantes con habilidades diferentes que participaron en este estudio, siendo el ámbito biológico donde se aplica la resiliencia. Aun en circunstancias críticas para la salud los individuos resilientes destacan por el vigor y seguridad para superarlos y reintegrarse a su vida (Lugo-Márquez et al., 2016), el procurar una resiliencia actitudinal influye en la protección de las condiciones corporales para obtener una calidad de vida completa. Se ha reportado prevalencia de adicciones o trastornos psicológicos en individuos no resilientes (Theis, 2003), así como la incidencia específica de depresión (Turcaz \& Rubio, 2015); sin 
embargo, la inversión pública en salud ha probado ser clave en la implementación de políticas orientadas a la sostenibilidad, bienestar y satisfacción personal (OECD, 2017; PNUD, 2016). La resiliencia respecto del rubro salud permite definir mejoras a nivel físico y mental al facilitar un análisis a largo término de tales condiciones en una población (Younsi, 2015), pues los jóvenes resilientes demuestran menor incidencia de intentos suicidas favoreciendo su calidad de vida (Huamán \& Jáuregui, 2015).

El presente estudio se ha enfocado en una muestra del conjunto de estudiantes, por lo que siendo la resiliencia y calidad de vida constructos variables, las implicancias discutidas deben ser interpretadas con cautela en la población universitaria. Se propone que investigaciones del tema a futuro se dirijan a variables contextuales, como el cuerpo docente, técnico y administrativo, que al estar en contacto directo con la población en cuestión permitan advertir otros agentes involucrados en el fomento de variables psicológicas positivas; así como emplear una metodología de estudios de caso para abordar aspectos situacionales individuales que son cruciales para el desarrollo de resiliencia y, así alcanzar calidad de vida. La educación enfocada en la calidad de vida de los estudiantes que facilite el aprendizaje para alcanzar felicidad personal ante la adversidad (Omar et al., 2011; Saavedra et al., 2015) será determinante en la realización personal, pues se ha demostrado que a mayor resiliencia se accede a mayor calidad de vida.

\section{REFERENCIAS}

Aguaded, M., \& Almeida, N. (2016). La resiliencia del docente como factor crucial para superar las adversidades en una sociedad de cambios. Tendencias Pedagógicas, 28,167-180. Recuperado de ttps://revistas.uam.es/tendenciaspedagogicas/ article/viewFile/3831/5211

Alonso-Aldana, R., Beltrán-Márquez, Y., MáfaraDuarte, R., \& Gaytán-Martínez, Z. (2016). Relación entre rendimiento académico y resiliencia en una universidad tecnológica. Revista de Investigaciones Sociales, 2(4), 38-49. Recuperado de https://goo.gl/ZDNzNn
Asociación Americana de Psicología. (2014). El camino a la resiliencia. Recuperado de https:// www.apa.org/centrodeapoyo/resiliencia-camino

Ballesteros, A. (2015). Características resilientes en adultos jóvenes que trabajan y/o estudian que han sido diagnosticados como portadores del virus VIH (Tesis de pregrado). Disponible en el Repositorio Institucional de URLG.

Barudy, J., \& Dantagnan, M. (2011). La fiesta mágica $y$ realista de la resiliencia infantil. Barcelona: Gedisa.

Bastaminia, A., Rezaei, M., Rezaei, M., \& Tazesh, Y. (2016). Resilience and quality of life among students of Yasouj Estate. International Journal of Research in Humanities and Social Studies, 3(8), 6-11. Recuperado de http://www.ijrhss.org/ papers/v3-i8/2.pdf

Baker, C. (2017). Quantitative research designs. Massachusetts: Jones \& Bartlett Learning.

Cáceres, I., \& Ccorpuna, R. (2015). Estresores académicos y capacidad de resiliencia en estudiantes de segundo, tercero y cuarto año. Facultad de enfermería UNSA. Arequipa. 2015. (Tesis de pregrado). Disponible en el Repositorio Institucional de UNSA.

Calderón, M. (2016). Factores de protección en la capacidad de resiliencia de los adolescentes de la Fundación Ecuasol (Tesis de pregrado). Disponible en el Repositorio Institucional de UDLA.

Cantú-Martínez, P. (2015). Calidad de vida y sustentabilidad: una nueva ciudadanía. Ambiente y Desarrollo, 19(37), 09-21. doi: 10.11144/ Javeriana.ayd19-37.cvsn

Cazan, A., \& Truta, C. (2015). Stress, resilience and life satisfaction in college students. Revista de Cercetare Si Interventie Sociala, 48, 95108. Recuperado de http://www.rcis.ro/en/ currentisue/2153-stress-resilience-and-lifesatisfaction-in-college-students.html

Comisión Europea (2012, 3 de octubre). El planteamiento de UE sobre la resiliencia: aprender de las crisis alimentarias. Recuperado de http://euro pa.eu/rapid/press-release_MEMO12-733_es.htm 
Connor, K. M., \& Davidson, J. R. T. (2003). Development of a new resilience scale: The Connor-Davidson resilience scale (CD-RISC). Depression and Anxiety, 18(2), 76-82. doi: 10.1002/da.10113.

Cyrulnik, B. (2002). Los patitos feos. Barcelona: Gedisa.

Everly, G., McCormack, D., \& Strouse, D. (2012). Seven characteristics of highly resilient people: Insights from navy SEALs to the "greatest generation." International Journal of Emergency Mental Health, 14(2), 87-93. Recuperado de https://www.ncbi.nlm.nih.gov/pubmed/23350224

Faircloth, A. (2017). Resilience as a mediator of the relationship between negative life events and psychological well-being (Disertación doctoral). Disponible en Electronic Theses \& Dissertations.

Federación Española de Síndrome de Down. (2013). Formación para la autonomía y la vida independiente. Madrid: ZINC Soluciones Creativas. Recuperado de https:// www.sindromedown.net/wp-content/ uploads/2014/09/144L_guia.PDF

Fergus, S., \& Zimmerman, M. A. (2005). Adolescent resilience: a framework for understanding healthy development in the face of risk. Annu Rev Public Health, 26, 99-419. doi:10.1146/annurev. publhealth.26.021304.144357

Fletcher, D., \& Sarkar, M. (2011). Psychological resilience: A review and critique of definitions, concepts and theory. European Psychologist, 18(1), 12-23. doi: 10.1027/1016-9040/a000124

Fraga, G. (2015). Resiliencia: un concepto que abre cuestionamientos sobre su construcción $y$ desarrollo. Montevideo: Universidad de la República de Uruguay.

Gallesi, R., \& Matalinares, M. (2012). Resiliencia y rendimiento académico en estudiantes del 5to y 6to grado de primaria. Revista de Investigación en Psicología, 15(1), 181-201. Recuperado de http:// sisbib.unmsm.edu.pe/bvrevistas/investigacion_ psicologia/v15_n1/pdf/a11v15n1.pdf

García-Alandete, J. (2016). Afrontando la adversidad. Resiliencia, optimismo y sentido de la vida.
Colección de Cuadernos de Psicología 04. Tenerife: Latina. Recuperado de http://www. cuadernosartesanos.org/Psicologia/2016/cdp04.pdf

Gómez, B. (2010). Resiliencia individual y familiar. Bilbao. S.e.

González-Arratia, N., \& Valdez, J. (2013). Resiliencia: diferencias por edad en hombres y mujeres mexicanos. Acta de Investigación Psicológica, 3(1), 941-955. doi: 10.1016/S20074719(13)70944-X

Grimaldo, M. (2003). Escala de Calidad de Vida de Olson \& Barnes. (Manual técnico). Lima: Universidad San Martin de Porres.

Grimaldo, M. (2012). Calidad de vida en estudiantes de secundaria de la ciudad de Lima. Av. Psicol. 20(1), 89-102. Recuperado de http://www. unife.edu.pe/pub/revpsicologia/avances2012/ mariagrimaldo.pdf

Grotberg,E.(2006). ¿Quéentendemos por resiliencia?, ¿cómo promoverla?, ¿cómo utilizarla? Barcelona: Gedisa.

Harvey, J., \& Delfabbro, P. H. (2004). Psychological resilience in disadvantaged youth: A critical overview. Australian Psychologist, 39(1), 3-13. https://doi.org/10.1080/00050060410001660281

Hernández-Holguín, D., Sánchez, I., Páez, E., \& Montoya-Vásquez, E. (2016). El desarrollo personal de los jóvenes de Medellín, Colombia: más allá de las conductas de riesgo y de resiliencia. Cadernos de Saúde Pública, 32(11), 1-10. Recuperado de https://doi.org/10.1590/0102$311 \times 00103614$

Hu, T., Zhang, D., \& Wang, J. (2015). A metaanalysis of the trait resilience and mental health. Personality and Individual Differences, 76, 1827. Recuperado de http://dx.doi.org/10.1016/j. paid.2014.11.039

Huamán, J., \& Jáuregui, H. (2015). Relación entre el nivel de resiliencia e ideación suicida en jóvenes universitarios de la Universidad Nacional de Cajamarca (Tesis de maestría). Disponible en el Repositorio Institucional de UPAGU.

Instituto de HeartMath. (2014). Construyendo resiliencia personal. California: Autor. 
Recuperado de http://www.ccc.org.co/ file/2017/11/RESILIENCIA-BPR-GuideSpanish.pdf

Leiva, L., Pineda, M., \& Encina, Y. (2013). Autoestima y apoyo social como predictores de la resiliencia en un grupo de adolescentes en vulnerabilidad social. Revista de Psicología de la Universidad de Chile, 22(2), 111-123. doi: 10.5354/07190581.2013 .30859

López, E., Guevara, V., \& Quinteros, Z. (2016). Religiosidad y resiliencia en estudiantes de psicología de una universidad privada de Lima Este. Revista Científica de Ciencias de la Salud, 9(2), 26-31. Recuperado de: https://revistas.upeu. edu.pe/index.php/rc_salud/article/view/227

Lugo-Márquez, C., Guerrero-Mojica, N., CastañedaGuerrero, M., Gámez-Roque, N., MartínezGarcía, I., \& Padilla-Muñoz, J. (2016). Resiliencia y factores de riesgo en estudiantes universitarios al inicio de su formación profesional. Rev Enferm Inst Mex Seguro Soc, 24(3), 171-176. Recuperado de http://www.medigraphic.com/ pdfs/enfermeriaimss/eim-2016/eim163d.pdf

Luthar, S. S., Cicchetti, D., \& Becker, B. (2000). The construct of resilience: A critical evaluation and guidelines for future work. Child Development, 71(3), 543-562. https://doi.org/10.1111/14678624.00164

Masten, A. S. (2001). Ordinary magic. Resilience processes in development. Am Psychol, 56(3), 227-238. doi: 10.1037/0003-066X.56.3.227

Mateau, R., García, M., Gil, J., \& Caballer, A. (2009). ¿Qué es la resiliencia? Hacia un modelo integrador. Valencia: Universitat Jaume I. Recuperado de https://goo.gl/6s6JX6

Ministerio de Educación del Perú. (2017). Estadística de la Calidad Educativa. Recuperado de http:// escale.minedu.gob.pe/ueetendencias20002015

Mikulic, I., Crespi, M., Caruso, A., Elmasian, M., Albornoz, O., García, L., ... Giardina, E. (2011). Estudio del potencial resiliente y la calidad de vida percibida en estudiantes universitarios. Buenos Aires: Departamento de Publicaciones-Facultad de Psicología-UBA. Recuperado de https://www. aacademica.org/000-052/926.pdf
Monardes, C., Gonzáles-Gil, F., \& Soto-Pérez, F. (2011). Calidad de vida: concepto, características y aplicación del constructo. Revista Sujeto, Subjetividad Y Cultura, 2, 68-86. Recuperado de ww.researchgate.net/publication/235989330_ Calidad_de_vida_conceptos_caracteristicas_y_ aplicacion_del_constructo

Nava, M. (2012). La calidad de vida: análisis multidimensional. Revista Medigraphic, 11(3), 129-137. Recuperado de http://www.medigraphic. com/pdfs/enfneu/ene-2012/ene123c.pdf

Notter, M., MacTavish, K., \& Shamah, D. (2008). Pathways toward resilience among women in rural trailer parks. Family Relations. Interdisciplinary Journal of Applied Family Science, 57(5), 613624. doi: 10.1111/j.1741-3729.2008.00527.x

Novella, A. (2002). Incremento de la resiliencia luego de la aplicación de un programa de psicoterapia breve en madres adolescentes (Tesis de maestría). Disponible en el Repositorio Institucional de UNMSM.

Obando, O., Villalobos, M., \& Arango, S. (2010). Resiliencia en niños con experiencias de abandono. Acta Colombiana de Psicología, 13(2), 149-159. Recuperado de https://goo.gl/bYHB79

Olson, D., \& Barnes, H. (1982). Calidad de vida. Manuscrito no publicado.

Omar, A., Paris, L., Uribe, H., Ameida, S., \& Aguiar, M. (2011). Un modelo explicativo de resiliencia en jóvenes y adolescentes. Psicologia Em Estudo, 16(2), 269-277. Recuperado de https://doi. org/10.1590/S1413-73722011000200010

Ong,A.D., Bergeman, C. S., Bisconti, T.L., \& Wallace, K. A. (2006). Psychological resilience, positive emotions, and successful adaptation to stress in later life. Journal of Personality and Social Psychology, 91(4), 730-749. doi: 10.1037/00223514.91.4.730

Organisation for Economic Co-operation and Development. (2017). How's life? 2017. S.1.: OECD Publicaciones.

Organización de Cooperación para el Desarrollo Económico. (2016). Making development happen. Avanzando hacia una mejor educación para Perú. S.1.: OCDE Publicaciones. 
Organización de las Naciones Unidas. (2015). Transformar nuestro mundo: la Agenda 2030 para el desarrollo sostenible (Reporte $\mathrm{N}^{\circ} \mathrm{A} / 70 / \mathrm{L} .1$ ). Recuperado de https://undocs.org/es/A/RES/70/1

Organización de las Naciones Unidas. (2016). Marco de cooperación de las Naciones Unidas para el desarrollo en Perú. Lima: ONU Publicaciones.

Pereira, R. (2007). Resiliencia individual, familiar y social. Recuperado de https://psiquiatria.com/ bibliopsiquis/resiliencia-individual-familiar-ysocial/

Pérez-Escoda, N. (2013, septiembre). Variables predictivas de la satisfacción con la vida en estudiantes universitarios. Comunicación presentada al XVI Congreso Nacional / II Internacional Modelos de Investigación Educativa de AIDIPE. Recuperado de https://goo. $\mathrm{gl} / \mathrm{Es} 1 \mathrm{GQM}$

Programa de las Naciones Unidas para el Desarrollo. (2014). Informe sobre Desarrollo Humano 2014. Sostener el progreso humano: reducir vulnerabilidades y construir resiliencia. Washington D.C.: Communications Development Incorporated. Recuperado de http://hdr.undp.org/ sites/default/files/hdr14-summary-es.pdf

Programa de las Naciones Unidas para el Desarrollo. (2016). Informe sobre Desarrollo Humano 2016: desarrollo humano para todas las personas. Washington D.C.: Communications Development Incorporated. Recuperado de http://hdr.undp.org/ sites/default/files/hdr_2016_report_spanish_web. pdf

Ruiz-Román, C., Calderón-Almendros, I., \& Juárez, J. (2017). La resiliencia como forma de resistir a la exclusión social: un análisis comparativo de casos. Pedagogía Social. Revista Interuniversitaria., 20(29), 129-141. Recuperado de https://doi. org/10.5565/rev/athenea.1697

Saavedra, E., Salas, G., Cornejo, C., \& Morales, P. (2015). La Psicología Educacional en diálogo con otras disciplinas. Talca: Universidad Católica del Maule.

Salazar,M.(2017).Optimismoyfelicidad enestudiantes de psicología de una universidad privada de Lima metropolitana (Tesis de pregrado). Disponible en el Repositorio Institucional de UPN.

Sambrano, J. (2011). Resiliencia. Transformación positiva de la adversidad. Ciudad de México: Alfa.

Seligman, M., \& Czikszentmihalyi, M. (2000), Positive Psychology: An Introduction. American Psychologist, 55(1), 5-14. doi: 10.1037/0003066X.55.1.5

Servicio Nacional de Aprendizaje. (2010). Proyecto de vida. Colombia: S.e.

Shaughnessy, J., Zechmeister, E., \& Zechmeister, J. (2012). Research methods in psychology. Nueva York: Mc Graw Hill.

Theis, A. (2003). La resiliencia en la literatura científica. Barcelona: Gedisa.

Torres, M., \& Ruiz, A. (2013). Habilidades de resiliencia en estudiantes de educación media superior del Estado de México. En Gaxiola, J., \& Palomar, J. (Eds.), Estudios de resiliencia en América Latina, 53-69. México: Pearson.

Turcaz, M., \& Rubio, Y. (2015). Intento suicida en adolescentes: factores resilientes y de riesgo. Revista de Información Científica, 93(5). Recuperado de http://www.revinfcientifica.sld.cu/ index.php/ric/article/view/181

Unión Europea. (2015). EuroStat. Luxemburgo: Unión Europea Publicaciones.

Velasco, V., Suárez, G., Córdova, S., Luna, L., \& Mireles, S. (2015). Niveles de resiliencia en una población de estudiantes de licenciatura y su asociación con variables familiares y académicas. Revista Iberoamericana de Producción Académica y Gestión Educativa, 2, 1-23. Recuperado de http://www.pag.org.mx/index.php/PAG/article/ view/490/529

Villasmil, J. (2010). El autoconcepto académico en estudiantes universitarios resilientes de alto rendimiento: un estudio de casos (Tesis doctoral). Disponible en el Repositorio Institucional de ULA.

Villasmil, J. (2013). Educación, gerencia de vida y rasgos psicosociales protectores del desarrollo 
en universitarios resilientes: un estudio de casos. Revista Arbitrada del Centro de Investigación y Estudios Gerenciales A.C., 4(1), 193-209. Recuperado de https://goo.gl/G57PLR

Wagnild, G., \& Young, H. (1993). Development and psychometric evaluation of the Resilience Scale. Journal of Nursing Measurement, 1(2), 165178. Recuperado de https://sapibg.org/.../1054wagnild_1993_resilience_scale_2.pdf

Yehuda, R., Daskalakis, N., Desarnaud, F., Makotkine, I., Lehrner, A., Koch, E., ... Bierer, L. (2013).
Epigenetic biomarkers as predictors and correlates of symptom improvement following psychotherapy in combat veterans with PTSD. Frontiers in Psychiatry, 4(118). doi: 10.3389/ fpsyt.2013.00118

Younsi, M. (2015). Health-related quality-of-life measures: Evidence from Tunisian population using the SF-12 Health Survey. Value in Health Regional Issues, 7, 54-66. Recuperado de https:// doi.org/10.1016/j.vhri.2015.07.004

Fecha de recepción: 13 de mayo 2020

Fecha de aceptación: 09 de junio 2020 